\title{
Il viaggio nel sogno del Polifilo: tra il locus horridus e il locus amoenus
}

\section{Abstract}

\section{The Oneiric Travel of Polifilo: Between Locus Horridus and Locus Amoenus}

The aim of this paper is to propose a lecture of some initial passages of Hypnerotomachia Poliphili, written by Francesco Colonna and published in 1499. First, we try to go through the tradition of descritpio locorum in ancient rhetoric treatises and the literary production, with respect to two frequent topoi: locus amoenus and locus horridus. We also follow their formal modifications in the Middle Ages, as Colonna draws both from the ancient and medieval tradition in its own construction of loci. Our premise is that the place description in the Colonna's opera, apart from being an important support for the narrative thread, plays an important structural role by ordinating events and helping that way their memorization. Moreover, the setting of imagery elements of the described places offers a clear interpretation key to the allegorical journey of Polifilo.

Keywords: Hypnerotomachia Polifili, oneiric travel, Polifilo, locus horridus, locus amoenus

L'opera di Francesco Colonna, Hypnerotomachia Poliphili, pubblicata a Venezia nel 1499, è un fenomeno unico sul mercato editoriale del Rinascimento italiano. Edita dalla casa di Aldo Manuzio, contiene quasi 200 xilografie preziose, illustrazioni al testo che lo completano e commentano. Fu molto popolare ai suoi tempi, non solo per i disegni, che furono considerati piccole opere d'arte, tanto da essere erroneamente attribuiti addirittura a fra Giocondo ${ }^{1}$. Le ragioni dell'insolito interesse che l'opera desta fino ad oggi vanno cercate nella complessità dell'opera nella quale, accanto al filo conduttore del viaggio onirico-sapienziale-amoroso di Polifilo si trovano innumerevoli riferimenti filosofici, archeologici, botanici,

${ }^{1}$ C. Huelsen, Le illustrazioni della „Hypnerotomachia Polliphili” e le antichità di Roma, edito a Firenze nel 1910, qui citato da: G. Contessi, Architetti-pittori e pittori-architetti, Bari 1985, n. 13, p. 83. 
i quali costituiscono un notevole contributo alla creazione di una vera e propria enciclopedia del sapere.

L'opera è composta di due libri, di cui nel primo si racconta del viaggio di Polifilo in cerca della sua amata Polia. Polifilo, svegliatosi nel sogno scopre di trovarsi in un luogo deserto e inizia un cammino il quale, dopo aver superato vari pericoli, lo porta a un esaurimento estremo. Così, si addormenta nuovamente e in questo secondo livello del sogno inzia veramente suo il viaggio psicoerotico--sapienziale, animato dall'aegritudo amoris - la malattia e l'arsura d'amore, topos di tradizione antica diffusosi nel Medioevo, il quale nell'Hypnerotomachia ha anche un altro lato - quello del desiderio di conoscenza. Per appagarlo, Polifilo deve attraversare molti luoghi, affrontare varie difficoltà e tentazioni, imparare a seguire la via di mezzo, ovvero il principio dell'aurea medietas. Solo nel momento in cui raggiunge un certo equilibrio e impara a dominare i sensi, incontra la sua amata Polia (ella stessa simbolo della saggezza) e arriva insieme a lei nel regno di Venere, il quale si trova nell'isola di Citera. Lì i due giovani vengono uniti in matrimonio. Il primo libro finisce con la richiesta rivolta da ninfe a Polia di raccontare la sua storia. Il secondo libro è proprio il racconto di Polia che parla della sua provenienza e dell'incontro con Polifilo. Polia racconta il proprio cambiamento: inizialmente impassibile all'amore di Polifilo, modifica il suo atteggiamento sotto l'influsso di varie visioni. La benedizione di Venere ancora una volta conclude la storia, ma nel momento in cui Polifilo vuole abbracciare l'amata, questa svanisce e Polifilo si sveglia e capisce che è morta.

Tutto quello che Polifilo incontra durante il suo cammino: luoghi, persone, numerosi monumenti antichi, ha un significato simbolico e riflette la sua condizione. I monumenti incontrati (piramide, colosso, elefante, cavallo, porta), elementi architettonici, infine la natura - hanno valore simbolico e rimandano alla tradizione mitologica e letteraria antica e medievale da una parte, fornendo elementi dell'immaginario, e alla filosofia e scienza dall'altra parte, le quali offrono la chiave interpretativa delle immagini. Se aggiungiamo ancora il simbolismo della luce, "veicolo principe della visione onirica" di Polifilo ${ }^{2}$, di chiara provenienza neoplatonica, ci troveremo di fronte a un'opera densa di significati che si intrecciano e si completano a vicenda.

Fra tutta questa ricchezza di motivi e risvolti interpretativi vorrei scegliere uno: la descrizione dei luoghi che accompagna e crea lo sfondo dell'azione e della trama. Volgere uno sguardo sulla costruzione paesaggistica del romanzo sembra rilevante giacché essa offre una struttura portante allo sviluppo del viaggio nel sogno del protagonista. I frammenti scelti per l'analisi mettono in risalto il primo momento in cui il protagonista inizia a liberarsi dai vincoli dei sensi che lo legano al corpo (entrando nelle due fasi del sogno) e a prendere coscienza del fatto che solo seguendo la regola del festina lente sarà in grado di arrivare al desiderato fine del viaggio. Avendo a disposizione uno spazio limitato dedicato a questo articolo,

${ }^{2}$ M. Gabriele, Festina tarde: sognare nella temperata luce dell'immaginazione [in:] Storia della lingua e storia dell'arte in Italia. Dissimmetrie e intersezioni, Atti del III Convegno ASLI Associazione per la Storia della Lingua Italiana (Roma, 30-31 maggio 2002), a cura di V. Casale e P. D’Achille, Roma 2004, p. 168. 
mi sono decisa a scegliere solo due frammenti iniziali del primo libro dell'opera, a mio avviso abbastanza importanti.

\section{La tradizione della descriptio locorum}

Naturalmente, l'idea di inserire descrizioni di luogo non fu un'invenzione colonniana. Come in tanti altri casi, l'autore attinge l'idea dalla ricchissima tradizione antica e medievale, senza però essere vincolato dalla convenzione. Prima di passare ai loci dell'Hypnerotomachia sembra fondato quindi avvicinare brevemente la storia della descriptio locorum precedente all'opera.

Probabilmente la descrizione del luogo accompagna l'uomo da sempre, essendo essa una naturale reazione verbale al contatto con la natura. Nella letteratura è presente da sempre, acquisendo una funzione para-idiomatica, spesso introducendo il racconto quasi come nell' apertura convenzionale delle favole „C'era una volta..."3. Mentre la trasfigurazione dei luoghi naturali descritti inizia con Omero, la teorizzazione della loro descrizione si deve soprattutto a Cicero (De inventione, I) e a Quintiliano (Institutio oratoria, III) i quali la collocano nella parte dell'inventio, dandone istruzioni particolari sugli argomenti per i quali un luogo può essere lodato o vituperato, sempre in corrispondenza all'azione umana a cui si accompagna nell'oratoria giudiziaria (dai tempi di Aristotele). I retori trattavano i rispettivi luoghi descritti come supporto alla memoria, la quale doveva sostenere il peso di un ampio e ordinato materiale del discorso. Così, la disposizione dei luoghi e la loro corrispondenza al carattere dell'azione umana costituivano una sorta di strumento mnemotecnico ${ }^{4}$. Vi si possono percepire anche le ragioni della regola del decorum, formulata da Cicerone e vigente nella letteratura fino ai tempi moderni. Un'altra motivazione a questa regola è di tipo psicologico: il luogo rifletteva le emozioni del protagonista, la sua vita interiore, l'atmosfera generale di ciò che accadeva, molto spesso offrendo addirittura un'interpretazione, cosa che si può osservare in numerosi esempi della letteratura antica e medievale, come le Metamorfosi ovidiane o la Commedia dantesca, per enumerare solo esempi di spicco.

Se vogliamo esaminare da vicino la struttura del topos, occorre scinderlo in due categorie: il locus amoenus, più conosciuto e meglio descritto, e il locus horridus, dalla funzione contrapposta al primo.

Il locus amoenus di regola è composto dai seguenti elementi: un prato con erba e fiori, l'ombra garantita da uno o più alberi e l'acqua corrente di un ruscello o meglio ancora - una fonte che sgorga in vicinanza. Si può sentire il canto degli uccelli, eventualmente l'aria mossa dai soffi di zefiro. In alternativa al prato, il locus può essere rappresentato da una caverna con gli stessi attributi. La funzione

3 D. Evett, ,Paradice's Only Map”: The „,Topos ” of the „Locus Amoenus” and the Structure of Marvell's „,Upon Appleton House”, „PMLA”, Vol. 85, No. 3, May 1970, p. 505.

4 N.W. Bernstein, Locus Amoenus and Locus Horridus in Ovid's Metamorphoses, „Wenshan Review of Literature and Culture", Vol 5 (1), December 2011, p. 72. 
di base di tale luogo è offrire una pausa narrativa, creare un'atmosfera di riposo, tranquillità, evasione, preparare uno sfondo alle vicende amorose.

Come si è detto, con Omero il mondo naturale che appare nella letteratura inizia ad acquisire caratteristiche particolari. È abitato dalle divinità: ninfe (Ill., XX, 8; Od., VI, 124 i XVII, 205) o Atene (Od., VI, 291), rappresentato da luoghi pacifici e fertili, come il giardino di Alcinoo (Od., VII, $112 \mathrm{sgg}$.), in cui regna un'eterna primavera. Dalla stessa atmosfera sono pervasi i Campi Elisi (Od., IV, 560-570), ai confini del mondo a cui dovrà recarsi Menelao, compiuto il corso della sua vita. Gli alberi, come il faggio (Ill. V, 237, 693; IX, 70), il fico (VI, 432; XI, 167) o il platano (II, 307) segnalano invece scene epiche.

La tradizione letteraria ha attinto da Omero gli elementi del paesaggio idillico. Sotto un platano oppure un faggio i poeti amavano riposare e comporre, spesso gareggiando. Il pastore diventa una figura emblematica del poeta e cantore. Nel III sec. a.C. nasce così, con Teocrito e i suoi Idilli, la poesia bucolica ${ }^{5}$. La descrizione emblematica del locus amoenus, quella dell'Idillio VII, intitolato Talisie, contiene tutti gli elementi costitutivi della tradizione del topos (VII, $135 \mathrm{sgg}$ ). È creata secondo il modello dell'isola di Sicilia, la terra nativa del poeta, grazie al che conserva un realismo caratteristico di tutta la tradizione da Omero a Virgilio, presente anche in un altro tipo di loco, introdotto da Teocrito nell'Inno a Dioscuri (XXII, $36 \mathrm{sgg}$.), ovvero la valle di Tempe (nella provincia greca di Tessalia). In questo caso abbiamo la combinazione di una splendida valle fluviale con una profonda gola montagnosa, sopra la quale si ergono montagne maestose. Appare qui anche una selva oscura e selvaggia, così cara ai loci medievali. Il topos passò poi nella tradizione retorica e letteraria. Fra molti poeti che lo adoperano troviamo Virgilio, il quale menziona la „frigida Tempe” nelle Georgiche (II, 467). Virgilio propose comunque un'altra locazione del paesaggio ameno: nell'Arcadia, un amalgama del Peloponesso greco e di Mantova. La descrizione emblematica del luogo ideale si trova nella prima delle sue Ecloghe. Bisogna tenere in mente però che sia in Teocrito che in Virgilio le descrizioni dei luoghi non sono molto elaborate, svolgono piuttosto una funzione di sfondo nella poesia bucolica. Gli elenchi degli alberi sono schematici e non hanno molto a che fare con la realtà. Lo scopo dell'inserimento di un locus amoenus è sempre quello di unire l'uomo alla natura. In questa prospettiva una particolare attenzione va dedicata a Ovidio, contemporaneo di Virgilio, i cui paesaggi diventano partecipi della narrazione. Nelle Metamorfosi (X, $86 \mathrm{sgg}$.) il bosco cresce miracolosamente sotto l'influsso della musica di Orfeo. Non di rado i personaggi del poema diventano parte del paesaggio, un'interazione tra l'uomo e la natura ben più lontana da quella prevista dalle rappresentazioni "standard" del locus amoenus. Così, le Eliadi furono trasformate in pioppi e Dafne in alloro, due alberi molto presenti nei paesaggi ovidiani. Nelle Metamorfosi il locus amoenus diventa spesso testimone di azioni violente ${ }^{6}$. Non è più un luogo di pace e riposo, ma che ammalia con promesse infrangendole su-

${ }^{5}$ E.R. Curtius, Literatura europejska i tacińskie średniowiecze, transl. A. Borowski, Kraków 2005, p. 195.

${ }^{6}$ N.W. Bernstein, op. cit., p. 76. 
bito dopo ${ }^{7}$. La funzione del topos cambia e sfuma nella direzione dell'altro, quello di locus horridus. Tale manipolazione delle aspettative del lettore familiare con la tradizione retorica dei topoi non era estranea ai poeti dell'epoca augustea.

Poco più tardi la descriptio locorum diventa oggetto di una decrizione molto elaborata. Uno degli esempi più belli si trova in Tiberiano, poeta latino del IV sec. Le sue immagini, tipiche della tarda Antichità, sembrano impressionistiche, ma come osserva Curtius ${ }^{8}$ realizzano in realtà delle linee guida precise dettate nel IV sec. d.C. da Libanio. Tutt'altro quindi che un impressionismo poetico. Tale approccio schematizzato venne maggiormente approfondito nell'epoca successiva.

Nel Medioevo il topos acquista la funzione allegorica. L'epoca eredita da Cicerone le cicrostanze dell'azione come elementi costitutivi della descriptio locorum (cfr. Marziano Capella, Liber de arte rhetorica), ma con Prisciano li abbandona. Il luogo acquista infatti una posizione autonoma rispetto all'azione umana, della cui - dal quel momento in poi-avrebbe costituito al massimo uno sfondo?. Il locus amoenus diventa un paesaggio mentale, non realistico, e le associazioni alla fertilità ed erotismo danno più volte occasione alla sua esegesi nei termini di ambiguità morale. La dimensione morale si nota anche nell'interpretazione cristiana dell'albero del locus amoenus come l'albero della Croce, come anche nell'interpretazione del locus intero come rifugio del peccatore, ma anche luogo dello smarrimento morale, in cui regna la sensualità ${ }^{10}$. I Campi Elisi di Virgilio venivano rielaborati dai poeti Cristiani per rappresentare il Paradiso, con l'aggiunta degli alberi da frutto (assenti nell'Eliseo), un'associazione immancabile al frutto proibito ${ }^{11}$. Anche l'epica filosofica incluse il locus al proprio repertorio topico e lo sviluppò nelle rappresentazioni del paradiso terrestre, come nell'Anticlaudianus di Allain de Lille, dove la Natura abita in un castello con torri in mezzo a un bosco ${ }^{12}$. Con il romanzo cortese, a partire dal XII sec., gli elementi epici del paesaggio si estendono ed evolvono. Uno dei motivi chiave qui, è la selva selvaggia attraversata dai cavallieri, in mezzo alla quale si trova un locus amoenus sotto forma di frutteto, come succede nel Roman de Thèbes o nel Cantar de Mio Cid. Anche Andrea Calleppano nel trattato De Amore menziona più volte un luogo ameno chiuso nella foresta, con attrubuti tipici del topos. La sua suggestiva descrizione delle tre parti chiamate Siccità, Umidità e Amenità (Am., I, 6E, 250 sgg.) costituisce uno schema ritrovabile nei giardini di Citera dell'Hypnerotomachia ${ }^{13}$.

Per quanto concerne il locus horridus, esso si può considerare come nozione antitetica al locus amoenus. Realizzando la stessa regola del decorum, costituisce lo sfondo alle azioni tristi o tragiche. I due topoi sono complementari nel senso

7 Ibid., p. 83.

${ }^{8}$ E R. Curtius, op. cit., p. 204.

9 T. Michałowska, Między poezja a wymowa. Konwencje i tradycje staropolskiej prozy nowelistycznej, Wrocław 1970, p. 161.

${ }^{10}$ D. Evett, op. cit., pp. 506-507.

${ }^{11}$ E.R. Curtius, op. cit., p. 206.

12 Ibid., p. 204.

13 A. Klimkiewicz, Hypnerotomachia Poliphili Francesca Colonny, Kraków 2015, pp. 110-112. 
che possono essere analizzati secondo gli stessi elementi costituenti: quelli che nel locus amoenus sono positivi, nel locus horridus o mancano del tutto oppure attirano l'attenzione con la loro bruttezza. È l'immagine di un luogo esteticamente sgradevole, che provoca tormento nell'uomo, minacciando la sua salute e la vita stessa. Provoca la sensazione di smarrimento e di terrore paralizzante. Non di rado diventa anche luogo d'incontro con il mondo ultraterreno. Nell'Antichità le descrizioni si rifacevano a immagini mitologiche del regno delle tenebre, con i motivi del fiume oscuro dall'acqua immobile, della palude, della terra deserta, tenebrosa e fredda, dei venti ghiaccianti, delle rocce ripide e piene di fessure ${ }^{14}$. In Omero, la terra dei Cimmeri nel Caucaso (Odissea, XI, 14-19) è avvolta in una perenne nebbia, nella quale non arriva mai il sole e che è un suolo limite in cui il mondo dei vivi incontra quello dei morti. Altri esempi dei loci horridi sono rintracciabili nell'Eneide di Virgilio: la porta al Dite e la discesa al Tartaro con il Fiume Acheronte, il Tartaro stesso, la caverna di Sibilla.

Ma il locus horridus può anche riflettere il carattere della figura ivi incontrata, come nelle Metamorfosi di Ovidio: mentre la narrazione dell'opera è di solito ambientata in un generico locus amoenus, l'altro locus corrisponde al personaggio che lo abita. È così con le figure di Fames, Somnus, Invidia e Fama. Somnus (Met., XI, 592-607), ad esempio, abita in una caverna che sprofonda in un silenzio assoluto e in una fitta nebbia che lo avvolge. Questa descrizione è ispirata direttamente a quella della terra dei Cimmeri dell'Odissea (XI, 14-19). È una diretta contrapposizione del solido procedimento descrittivo del locus amoenus, in questo caso attraverso un catalogo di assenze. Mancano quindi: la luce del sole, paesaggi ameni, vegetazione, canto degli uccelli ${ }^{15}$.

Nella successiva produzione cristiana le immagini si incentrano sulle visioni dell'inferno o del purgatorio, con il locus horridus più elaborato nella storia della letteratura: l'inferno dantesco. Una varietà delle immagini topiche fu sviluppata anche dall'agiografia medievale, specialmente dalla letteratura partistica, nella quale appaiono descrizioni di luoghi deserti e sassosi e grotte fredde e buie che servono da dimore ai dediti alla vita religiosa ${ }^{16}$.

Bisogna tener presente che in ogni epoca vi furono autori più o meno creativi: questi ultimi adoperavano il topos in modo automatico, proprio come un idioma, quelli primi lo rimaneggiavano creativamente. Colonna si trova senz'altro fra quelli capaci di sfruttare la tradizione per i propri obiettivi.

\section{La costruzione per loci}

L'autore dell'Hypnerotomachia si serve volentieri del paesaggio in funzione dello sfondo narrativo. Esso accompagna momenti cruciali del cammino di Polifilo, preannunciandoli o riassumendoli in qualche modo, a volte commentandoli simbolicamente. Gli elementi canonici dei loci, come l'acqua, l'ombra, la pianura o il

\footnotetext{
14 T. Michałowska, op. cit., p. 203.

15 N.W. Bernstein, op. cit., p. 89.

16 T. Michałowska, op. cit., p. 203.
} 
bosco servono a tale scopo. Ad un'attenta lettura si possono scorgere descrizioni che sembrano formare dei patterns of meaning, secondo la terminologia di Richard Lansing che li aveva notati nelle similitudini della Commedia dantesca ${ }^{17}$. La struttura di tali serie è simile: la sequenza degli elementi è analoga, si notano invece differenze nella rappresentazione di alcuni particolari dell' immagine dipinta. Azzarderei l'ipotesi che l'analogia strutturale si rifaccia non solo sulla tradizione della descriptio locorum, ma che sia anche uno strumento mnemotecnico per facilitare la memorizzazione dell'intero racconto. Invece le differenze nell'impostazione dei particolari della scena servirebbero a evidenziare la condizione del protagonista la quale muta durante il viaggio.

In seguito si tenterà una lettura, in questa prospettiva, di alcuni frammenti di descrizioni del luogo tratti dall'inizio del romanzo: a partire dal primo locus amoenus a p.13, passando per due sequenze dei loci che sembrano parallele, fino alla p. 75 del primo libro dell'Hympnerotomachia Poliphili. Come menzionato sopra, la scelta di questi due frammenti dell'inizio dell'opera è dettata dall'intenzione di mettere in risalto un primo momento dell'evoluzione del protagonista: dalla condizione dell'incapacità di compresione e del senso di smarrimento nei sensi alla prima lezione di temperanza, sempre più presente nel suo viaggio e grazie alla cui dominazione sarà in grado di raggiungere la meta desiderata. È doveroso tuttavia menzionare che non è stato facile estrapolare dal romanzo passaggi isolati in quanto nell'opera ogni passaggio sembra intrecciarsi a tanti altri formando un insieme indivisibile.

Non avendo abbastanza spazio a disposizione per citare in extenso i brani inclusi nella presente ricerca, ne presento i riassunti contenutistici. La situazione descritta alle pagine 13-19 dell'Hypnerotomiachia è questa: Polifilo, dopo essersi addormentato, si trova nel sogno in una pianura deserta e quieta. La planitie è un luogo di verità e di contemplazione, secondo l'interpretazione quattrocentesca di questo elemento indispensabile del paesaggio „tipizzato”, i cui precedenti si ritrovano già nell'Odissea e nell'Eneide ${ }^{18}$. Per Polifilo, il silenzio della pianura è privo di significato perché non è capace di dedicarsi alla contemplazione, essendo ancora dominato dai sensi. Così, rivolge i passi verso una selva vicina, nella quale si perde dopo aver smarrito la strada. Il binomio: pianura-selva è noto nella letteratura, specialmente in quella medievale (Dante e soprattutto Boccaccio). La selva appare come simbolo dello smarrimento morale e del peccato, ma nel romanzo cavalleresco spesso conduce il viandante a un luogo ameno in mezzo al bosco (cfr. Geoffroi de Vinsauf, Ars versificatoria e Cappellano, De amore). Si aggiunga anche l'interpretazione di matrice platonica dell'antitesi anima-corpo, in cui l'anima è il viaggiatore e il corpo la selva dei sensi ${ }^{19}$. Nell' $H P$ la selva si configura comunque come un tipico locus horridus: è un luogo ostile, buio, che provoca terrore nel protagonista e non lo porta a nessuna meta pacifica, anzi, lo "attacca" strappandogli i vestiti con le spine dei cespugli tra i quali egli cerca di

${ }_{17}$ R. Lansing, From Image to Idea: A Study of the Simile in Dante's Commedia, Ravenna 1977.

${ }_{18}$ F. Colonna, Hypnerotomachia Poliphili, a cura di Marco Ariani e Mino Gabriele, Milano 2004, Vol. 2, pp. 520-521, n. 1.

${ }^{19}$ Ibid., pp. 520-521, n. 1. 
farsi strada ${ }^{20}$, inciampando continuamente. Solo dopo l'invocazione al padre della luce, Giove, riesce ad uscire in un bosco diradato dove incontra un ruscello con acqua fresca, ma benché sul punto di morire dalla sete e dalla stanchezza, non vi si dissetta perché sente una bellissima voce e la segue, perdendo di vista l'acqua. La ragione per cui Polifilo non appaga il suo desiderio di bere, è da ricercarsi nell'interpretazione dell'acqua come fonte sapienziale: non la può bere finché non si sarà liberato dalla voluptas ${ }^{21}$. Invece la musica che lo porta lontano dalla selva-corpo e dai travagli del locus horridus è un elemento delle descrizioni dei loci amoeni presente già nella letteratura ellenistica e in quella cortese medievale (cfr. Roman de la Rose; Boccaccio, Amorosa Visione). Seguendola, Polifilo arriva in un luogo ameno, sempre deserto di gente e animali, dove si sdraia sotto una quercia e finalmente si riposa, entrando alla fine in un secondo sogno, in cui parte veramente per il suo viaggio onirico ${ }^{22}$. Il luogo in cui si addormenta si trova ancora nel primo livello del sogno. Probabilmente per questo non è in grado di raggiungere la fonte della musica, la quale è appena un'eco dell'aldilà che risuona nelle sue orecchie ${ }^{23}$. La quercia è un albero simbolico sacro a Giove, sotto il quale nell'Antichità si addormentava chi voleva ricevere una risposta divina al proprio quesito ${ }^{24}$.

Così, il protagonista trovandosi nell'anticamera del proprio viaggio sapienziale, non ha accesso a molte cose: acqua, la fonte del canto, ovvero, a livello simbolico: non è capace di comprendere significati di cose che gli si presentano perché non domina ancora i propri sensi.

Infatti, inizierà a imparare a dominarli più avanti, ammonito più volte dalle iscrizioni su diversi oggetti incontrati lungo il cammino. Nella sequenza successiva, analoga alla prima descritta sopra, Polifilo, ,svegliatosi” nel secondo sogno passa di nuovo per una pianura dolce e quieta (HP, p. 20 sgg.), ancora senza traccia della presenza degli animali né dell'uomo, ma questa volta circondata da soavi colline e graziosi boschetti. Questo locus amoenus lo conduce a una piramide nella quale entra attraverso una magnifica porta e, volendo tornare indietro, incontra un drago dal quale si rifugia nel labirinto sotterraneo della piramide. Il labirinto si configura come un altro locus horridus - simbolo della prigione corporale, del traviamento dei sensi, analogamente alla selva oscura del primo frammento. Il labirinto costituisce un passaggio da una dimensione a un'altra, una „morte simbolica [...] che l'anima deve superare per tornare nella patria perduta" ${ }^{25}$, la quale si può identificare con il luogo ameno trovato dopo l'uscita dal labirinto. È il passaggio dall'error alla patria celeste ritrovata e a un locus amoenus del capitolo seguente, questa volta dipinto come dimora di ninfe e dèi antichi ${ }^{26}$. Infatti, gli ele-

${ }^{20}$ Il fatto di strappare le vesti si richiama all'allegoria dell'antitesi corpo-anima, in cui il progressivo spogliarsi dalle vesti significa la purificazione dell'anima, la sua liberazione dai legami mondani per risalire al divino. F. Colonna, op. cit., Vol. 2, p. 529, n. 5.

${ }^{21}$ F. Colonna, op. cit., Vol. 2, p. 526-530, n. 5.

22 Ibid., pp. 537-538, n. 4.

${ }^{23}$ Ibid., p. 537, n. 4.

${ }^{24}$ Ibid., p. 540, n. 2.

${ }^{25}$ Ibid., pp. 663-664, n. 3.

${ }^{26}$ Ibid., p. 663, n. 3. 
menti del paesaggio che vi ritroviamo rientrano nello schema simbolico archetipico: il bosco, l'acqua, la montagna intorno. Anche qui lo scampo è reso possibile dopo una preghiera alle divinità, e come l'altra volta Polifilo esce alla luce del giorno completamente sfinito fisicamente e sconvolto psichicamente. Anche qui abbiamo un susseguirsi degli stati di ansia e di tranquillità.

Polifilo, uscito da una porta angusta vede un locus amoenus pieno di luce, solitario. Si trova su un monte. Scende e ai piedi di esso passa sotto una macchia di castagni con freschi e umidi prati e grate ombre. A questo punto scorge un ponte (HP, p. 69), sotto il quale scorre l'acqua che si divide in due ruscelli che alimentano una valle fiorita, incorniciata dai monti. Oltre a una folta vegetazione, vi sono uccelli, scoiattoli e ghiri. Il luogo non è quindi così silenzioso e deserto come la prima delle pianure incontrate, ma sempre privo di esseri umani.

In fondo alla valle vede un edificio, con una fontana che raffigura una ninfa dormiente. L'acqua che esce dai due capezzoli della ninfa (fredda dal destro, calda dal sinistro) e che sgoccia in un unico vaso simboleggia l'amore razionale. L'immagine figurativa è d'ispirazione antica, ma il suo significato allegorico è medievale: l'acqua fredda simboleggia l'amore per vano piacere, l'acqua calda - l'amore avaro e arido. Tutte e due si mescolano in un vaso di porfirite diventando acqua tiepida che alimenta una pianura fertilissima (cfr. il fonte d'amore: Boccaccio, Am. Vis. e Cappellano, Am.). La metafora si legge così: l'amore onesto, il medium virtuoso, costituisce il vero nutrimento del mondo, della Natura ${ }^{27}$. Ed è a quest' acqua che Polifilo disseta finalmente la sua arsura amorosa.

La lezione di sottomettere la passione alla ragione e saper procede sulla via di mezzo (l'aurea medietas), insegnatagli dalle scritte sul ponte (l'ammonimento alla temperanza e alla prudenza), permette a Polifilo di procedere e attraversare il ponte raggiungendo un altro locus amoenus.

La seconda sequenza dei luoghi si può riassumere così: nelle tenebre della piramide, e poi dalle iscrizioni geroglifiche sul ponte Polifilo apprende la saggezza del festina lente e dell'aurea medietas, ribadita poi dal simbolismo dell'acqua temperata della fonte ninfale. Inizia quindi a dominare i sensi corporali, è un punto di svolta nel suo cammino, a partire dal quale troveremo loci amoeni sempre più orditati dalla mano umana, abitati da ninfe e divinità. Il primo di tali loci è il viale alberato che porta alla reggia di Eleuterillide (HP, p. 88), l'ultimo - l'isola di Citera, alla quale arrivano Polifilo e Polia alla fine del primo libro ${ }^{28}$. Se consideriamo il valore simbolico del nome della donna amata da Polifilo: della ,luce sofianica" 29 , sembra plausibile l'interpretazione del viaggio amoroso del protago-

${ }^{27}$ La fonte è infatti dedicata alla „Madre di tutte le cose” HP 72. F. Colonna, op. cit., Vol. 2, pp. 666-670, n. 2; pp. 670-671, n. 3.

${ }^{28}$ Analisi dei frammenti menzionati si trova nell'articolo „Locus amoenus nell'Hypnerotomoachia Poliphili: strumento interpretativo del viaggio onirico sapienziale", in pubblicazione nel volume degli atti del Convegno „'gli uernacoli, proprii, et patrii uocabuli' - Terminologia e fonti dell'Hypnerotomachia Poliphili (Venezia 1499)", Villa Vigoni, 22-26.10.2018 (data della pubblicazione: 2020 ).

${ }^{29}$ F. Colonna, op. cit., Vol. 2, p. 683-686, n. 6. 
nista nei termini cognitivi, del passaggio dai sensi confusi e incontrollati allo stato di comprensione razionale e ordinata.

\section{Conclusioni}

Nella descriptio locorum dei passi scelti abbiamo avuto modo di notare molti topoi che presentano chiari e numerosi riferimenti intertestuali, sia alla letteratura antica che a quella medievale. Tali rimandi non sono un puro sfoggio di erudizione dell'autore: intraprendono un dialogo con la tradizione proponendo una soluzione unica e nuova. Il simbolismo delle immagini è di natura cognitiva e sapienziale e riflette la condizione della immaginatio del protagonista, la quale si trova in qualche modo appesa tra il corpo (i sensi) e l'intelletto. Solo sapendo trovare la strada di mezzo, l'aurea medietas che si nutre delle immagini corporali per produrre sensi intellettuali, il viaggio onirico riesce a giungere alla conclusione desiderata. Abbiamo presentato frammenti dell'opera in cui il protagonista, all'inizio dominato dalla corporeità e per questo perduto nella „selva dei sensi”, prende per la prima volta la via di mezzo e inizia a imparare la regola del festina lente. La trasformazione della sua condizione viene accompagnata dai mutamenti nei luoghi che attraversa. È proprio questa corrispondenza luogo-condizione interiore che ha attirato il mio interesse e che ho voluto esaminare.

Provando pertanto a trarre le somme dalla lettura dei due frammenti presentati sopra, si possono elencare alcuni punti su cui volgere l'attenzione. In primo luogo accennerei alla macrostruttura del romanzo: come si vede dall'analisi di questa sua parte iniziale, il carattere dei luoghi descritti è in diretta corrispondenza allo svolgimento delle vicende narrate, ma allo stesso tempo alla condizioneinterna del protagonista. Realizza quindi in pieno le prescrizioni ciceroniane della regola del decorum. D'altra parte una certa ripetizione dei motivi, la sequenza stessa degli elementi che rieccheggia nelle sequenze successive, facilita senz'altro la comprensione e l'orientamento nelle vicende narrate: svolge pertanto la funzione mnemotecnica raccomandata dagli antichi e applicata nelle opere letterarie della tradizione orale. L'altro lato di interesse è la microstruttura, ovvero l'immaginario dei luoghi descritti. Esso trae ispirazione dal mondo antico, o seguendo l'elenco degli elementi obbligatori topici del locus amoenus (ev. horridus) nelle descrizioni della natura oppure prestandone oggetti, come la piramide o la fontana della ninfa dormiente, piena di riferimenti antiquari. Il valore simbolico di questi elementi del paesaggio, e la risposta emozionale di Polifilo (terrore o senso di quiete e felicità) si confermano a vicenda. Il Medioevo, fornendo anch'esso elementi dell'immaginario, come ad es. la foresta attraversata dal protagonista, dentro alla quale si trova un locus amoenus, offre prima di tutto la chiave interpretativa. Infatti, l'interpretazione sia morale che psicoerotica di provenienza cortese sembra prevalere in molti frammenti dell'opera. Non possiamo dimenticare comunque che si tratta di una ,psicomachia d'amore” in cui i luoghi orridi e le tenebre simboleggiano le pene dell'aegritudo amoris, allo stesso tempo rappresentando tuttavia gli impedimenti che l'anima incontra nella sua visio in somnis, nel 
volo onirico ${ }^{30}$. In questo modo abile e sincretico il Colonna seppe unire nella sua opera i motivi classici e medievali, artistici e filosofici in un'unica reinterpretazione rinascimentale, in un ,[...] libro, che riesce a fondere [...] immaginario antico e immaginario medievale in una sintesi umanisticamente nutrita di filologia e antiquaria" 31 , come sostengono alcuni, addirittura di gusto barocco o preromantico.

\section{Bibliografia}

Bernstein N.W., Locus Amoenus and Locus Horridus in Ovid's Metamorphoses, „Wenshan Review of Literature and Culture", Vol 5 (1), December 2011, pp. 67-98.

Colonna F., Hypnerotomachia Poliphili, a cura di Marco Ariani e Mino Gabriele, Voll. 1, 2, Milano 2004.

Contessi G., Architetti-pittori e pittori-architetti, Bari 1985.

Curtius E.R., Literatura europejska i łacińskie średniowiecze, thum. A. Borowski, Kraków 2005.

Evett D., „Paradice's Only Map”: The „Topos” of the „Locus Amoenus” and the Structure of Marvell's „,Upon Appleton House”, „PMLA”, Vol. 85, No. 3, May 1970, pp. 504-513.

Gabriele M., Festina tarde: sognare nella temperata luce dell'immaginazione, in: Storia della lingua e storia dell'arte in Italia. Dissimmetrie e intersezioni, Atti del III Convegno ASLI Associazione per la Storia della Lingua Italiana (Roma, 30-31 maggio 2002), a cura di V. Casale e P. D’Achille, Roma 2004, pp. 161-174.

Klimkiewicz A., Hypnerotomachia Poliphili Francesca Colonny, Kraków 2015.

Lansing R., From Image to Idea: A Study of the Simile in Dante's Commedia, Ravenna 1977.

Michałowska T., Między poezją a wymowa. Konwencje i tradycje staropolskiej prozy nowelistycznej, Wrocław 1970.

Wergiliusz P.M., Eneida, tłum. T. Karyłowski, Wrocław 2004.

Wilhelm z Lorris, Jan z Meun, Powieść o róży, tłum. M. Frankowska-Terlecka, T. Giermak-Zielińska, Warszawa 1997.

\footnotetext{
30 M. Gabriele, op. cit.

31 Ibid., Vol. 2, Intr., p. LXI.
} 